\title{
Bispectrality for the quantum open Toda chain
}

\author{
E. K. Sklyanin
}

\begin{abstract}
An alternative to Babelon's (2003) construction of dual variables for the quantum open Toda chain is proposed that is based on the $2 \times 2$ Lax matrix and the corresponding quadratic $R$-matrix algebra.
\end{abstract}

\section{INTRODUCTION}

The term bispectrality [DG1986] refers to a situation when a function $f(x, \lambda)$ depending on two variables $x$ and $\lambda$ simultaneously satisfies two spectral problems: one being a differential or finite-difference equation $H\left(x, \partial_{x}\right) f=\lambda f$ in $x$, with $\lambda$ playing the role of the spectral parameter, and the dual one, $\widetilde{H}\left(\lambda, \partial_{\lambda}\right) f=x f$ in $\lambda$, with $x$ being the spectral parameter. The function $f(x, \lambda)$ can thus be considered as a kernel of an integral operator providing expansion in the eigenfunctions of the operator $H$, or of $\widetilde{H}$, depending on the point of view.

In the simplest examples, the bispectrality is a manifestation of the contiguity relations for the hypergeometric function.

In the context of multivariate quantum integrable systems, $x$ and $\lambda$ become sets of $N$ variables, and the two spectral problems become spectral problems for commuting quantum Hamiltonians. The bispectral formulation has been found for the quantum open Toda chain [Bab2003, Bab2004], the quantum Calogero-Moser system and its generalisations [Chal2000], the Knizhnik-Zamolodchikov equation [TV2002], Gaudin models [MTV2008], to name a few examples.

The bispectrality seems to be a common property enjoyed by multidimensional hypergeometric equations, among their other characteristic properties, like existence of integral representations for the eigenfunctions with the kernel expressed in terms of elementary functions, raising/lowering operators etc.

The purpose of this letter is to revise the case of the quantum open Toda chain and to suggest a few improvements to Babelon's [Bab2003, Bab2004] original construction of the dual variables for that model. Like Babelon, we start with the construction for the classical (non-quantum) case. However, unlike [Bab2003], we do not consider the periodic case that leads to difficult algebraic-geometric constructions for hyperelliptic curves. Instead, we start directly with the open Toda chain and use the observation of [KV2000] that the corresponding 
spectral curve is a rational algebraic curve. The resulting formulae are the same as in [Bab2003] but the derivation is simplified drastically.

In [Bab2003, Bab2004] the formulae for the quantum case were conjectured based on the results for the classical case. However, the proof of the conjectured formulae given in [Bab2003, Bab2004] does not follow the classical construction and is based instead on the integral representations for the eigenfunctions found in [KL2001]. Our derivation has an advantage that it is completely parallel to the classical case and is considerably simpler that in the pioneering papers [Bab2003, Bab2004]. Besides, being based on the $R$-matrix algebra for the monodromy matrix, it allows, in principle, generalisations to other integrable models.

\section{DESCRIPTION OF THE MODEL}

We start with a discussion of the classical case. In what follows we use the notation of [Skl1985, Skl2000].

The open $n$-particle Toda chain is described in terms of the canonical variables

$$
\left\{X_{j}, X_{k}\right\}=\left\{x_{j}, x_{k}\right\}=0, \quad\left\{X_{j}, x_{k}\right\}=\delta_{j k}, \quad j, k=1, \ldots, n .
$$

and is characterised by the Hamiltonian

$$
H=\sum_{j=1}^{n} \frac{1}{2} X_{j}^{2}+\sum_{j=1}^{n-1} \mathrm{e}^{x_{j+1}-x_{j}} .
$$

Defining local Lax matrices as

$$
\ell_{j}(u)=\left(\begin{array}{cc}
u+X_{j} & -\mathrm{e}^{x_{j}} \\
\mathrm{e}^{-x_{j}} & 0
\end{array}\right)
$$

we introduce the partial monodromy matrices

$$
\begin{aligned}
L_{j k}(u) & \equiv \ell_{j}(u) \ell_{j-1}(u) \ldots \ell_{k+1}(u) \ell_{k}(u)=\left(\begin{array}{cc}
A_{j k}(u) & B_{j k}(u) \\
C_{j k}(u) & D_{j k}(u)
\end{array}\right), \quad \begin{array}{l}
j \geq k, \\
L_{j k}
\end{array}=\left(\begin{array}{ll}
1 & 0 \\
0 & 1
\end{array}\right), \quad j<k,
\end{aligned}
$$

and the complete monodromy matrix

$$
L(u) \equiv L_{n 1}(u)=\ell_{n}(u) \ldots \ell_{2}(u) \ell_{1}(u)=\left(\begin{array}{cc}
A(u) & B(u) \\
C(u) & D(u)
\end{array}\right) .
$$

To study the open Toda chain it is sufficient to work with the single column-vector $A C$ :

$$
\left(\begin{array}{l}
A(u) \\
C(u)
\end{array}\right) \equiv\left(\begin{array}{l}
A_{n 1}(u) \\
C_{n 1}(u)
\end{array}\right)=L(u)\left(\begin{array}{l}
1 \\
0
\end{array}\right)
$$


defined recursively:

$$
\left(\begin{array}{l}
A_{j 1}(u) \\
C_{j 1}(u)
\end{array}\right)=\left(\begin{array}{cc}
u+X_{j} & -\mathrm{e}^{x_{j}} \\
\mathrm{e}^{-x_{j}} & 0
\end{array}\right)\left(\begin{array}{l}
A_{j-1,1}(u) \\
C_{j-1,1}(u)
\end{array}\right), \quad\left(\begin{array}{l}
A_{01}(u) \\
C_{01}(u)
\end{array}\right)=\left(\begin{array}{l}
1 \\
0
\end{array}\right)
$$

or, componentwise

$$
\begin{gathered}
A_{j 1}(u)=\left(u+X_{j}\right) A_{j-1,1}(u)-\mathrm{e}^{x_{j}} C_{j-1,1}, \\
C_{j 1}(u)=\mathrm{e}^{-x_{j}} A_{j-1,1}(u), \\
A_{j 1}(u)=\left(u+X_{j}\right) A_{j-1,1}(u)-\mathrm{e}^{x_{j}-x_{j-1}} A_{j-2,1}(u) .
\end{gathered}
$$

The generating function of the integrals of motion of the open chain

$$
\begin{gathered}
A(u)=u^{n}+H_{1} u^{n-1}+\ldots+H_{n} . \\
H_{1}=X_{1}+\ldots+X_{n}, \quad H_{2}=\frac{1}{2} H_{1}^{2}-H .
\end{gathered}
$$

can be considered as the limit as $\varepsilon \rightarrow 0$ of the quasiperiodic chain

$$
\begin{gathered}
t_{\varepsilon}(u)=\operatorname{tr} L K_{\varepsilon}=A(u)+\varepsilon D(u), \quad K_{\varepsilon}=\left(\begin{array}{ll}
1 & 0 \\
0 & \varepsilon
\end{array}\right), \\
A(u)=\operatorname{tr} L K_{0}, \quad K_{0}=\left(\begin{array}{ll}
1 & 0 \\
0 & 0
\end{array}\right),
\end{gathered}
$$

with the boundary condition (twist) determined by the matrix $K_{\varepsilon}$, when the hyperelliptic spectral curve

$$
\operatorname{det}(v-L(u))=v^{2}-t_{\varepsilon}(u) v+\varepsilon=0
$$

degenerates into a rational one

$$
v=A(u) \text {. }
$$

The observation that the spectral curve for the open Toda chain is rational is due to [KV2000] and plays the key role in our construction. Working directly with the rational curve rather with the functions on the hyperelliptic curves and then taking the limit $\varepsilon \rightarrow 0$ in the end, allows to simplify the derivations considerably.

The local Lax matrices (2.3) satisfy the $r$-matrix Poisson brackets relations

$$
\left\{\stackrel{1}{\ell}\left(u_{1}\right), \stackrel{2}{\ell}\left(u_{2}\right)\right\}=\left[r\left(u_{12}\right), \stackrel{1}{\ell}\left(u_{1}\right)^{\ell}\left(u_{2}\right)\right], \quad r(u)=\frac{\mathcal{P}_{12}}{u},
$$

where $u_{12} \equiv u_{1}-u_{2}$ and $\mathcal{P}_{12}$ is the permutation operator (see [Skl1985, Skl2000] for the explanation of the notation).

The relations (2.16) imply immediately the same relations for the monodromy matrices $L_{j k}(u)$

$$
\left\{\stackrel{1}{L}_{j k}\left(u_{1}\right), \stackrel{2}{L}_{j k}\left(u_{2}\right)\right\}=\left[r\left(u_{12}\right), \stackrel{1}{L}_{j k}\left(u_{1}\right) \stackrel{2}{L}_{j k}\left(u_{2}\right)\right],
$$

including the case $L(u) \equiv L_{n 1}(u)$. As a consequence,

$$
\left\{A\left(u_{1}\right), A\left(u_{2}\right)\right\}=\left\{C\left(u_{1}\right), C\left(u_{2}\right)\right\}=0,
$$




$$
\left\{A\left(u_{1}\right), C\left(u_{2}\right)\right\}=\frac{-A\left(u_{1}\right) C\left(u_{2}\right)+C\left(u_{1}\right) A\left(u_{2}\right)}{u_{1}-u_{2}} .
$$

The dual variables $\left(\lambda_{j}, \Lambda_{j}\right), j=1, \ldots, n$ are introduced by the equations

$$
\begin{array}{rlrl}
\lambda_{j}: & & A\left(\lambda_{j}\right) & =0, \\
\Lambda_{j}: & \Lambda_{j} & =C\left(\lambda_{j}\right),
\end{array}
$$

and one can verify that

$$
\left\{\Lambda_{j}, \Lambda_{k}\right\}=\left\{\lambda_{j}, \lambda_{k}\right\}=0, \quad\left\{\Lambda_{j}, \lambda_{k}\right\}=-\Lambda_{j} \delta_{j k}
$$

The $A C$ vector is reconstructed in terms of the dual variables through the interpolation formulae:

$$
\begin{aligned}
& A(u)=\prod_{j=1}^{n}\left(u-\lambda_{j}\right), \\
& C(u)=\sum_{j=1}^{n} \Lambda_{j}\left(\prod_{k \neq j} \frac{u-\lambda_{k}}{\lambda_{j}-\lambda_{k}}\right) .
\end{aligned}
$$

An alternative approach is based on the 'large' $n \times n$ Lax matrix instead of the $2 \times 2$ as above [Fl1974, Man1974].

The open chain is served by the Lax matrix without the spectral parameter $\left(x_{j k} \equiv x_{j}-x_{k}\right)$ :

$$
\mathcal{L}=\left(\begin{array}{ccccc}
-X_{1} & 1 & \ldots & 0 & 0 \\
\mathrm{e}^{x_{21}} & -X_{2} & \ldots & 0 & 0 \\
\ldots & \ldots & \ldots & \ldots & \ldots \\
0 & 0 & \ldots & -X_{n-1} & 1 \\
0 & 0 & \ldots & \mathrm{e}^{x_{n, n-1}} & -X_{n}
\end{array}\right)
$$

The spectral parameter for the quasiperiodic chain is introoduced by adding two one-dimensional projectors (rank 2 perturbation, ergo hyperelliptic curve):

$$
\begin{aligned}
\mathcal{L}(v) & =\left(\begin{array}{ccccc}
-X_{1} & 1 & \ldots & 0 & \varepsilon v^{-1} \mathrm{e}^{x_{1 n}} \\
\mathrm{e}^{x_{21}} & -X_{2} & \ldots & 0 & 0 \\
\ldots & \ldots & \ldots & \ldots & \ldots \\
0 & 0 & \ldots & -X_{n-1} & 1 \\
v & 0 & \ldots & \mathrm{e}^{x_{n, n-1}} & -X_{n}
\end{array}\right) \\
& =\mathcal{L}+v|g\rangle\left\langle f\left|+\varepsilon v^{-1}\right| \widetilde{g}\right\rangle\langle\widetilde{f}|,
\end{aligned}
$$

where

$$
\langle f| \equiv(1,0, \ldots, 0), \quad\langle\widetilde{f}| \equiv\left(0, \ldots, 0, \mathrm{e}^{-x_{n}}\right)
$$




$$
|g\rangle=\left(\begin{array}{c}
0 \\
\vdots \\
0 \\
1
\end{array}\right), \quad|\widetilde{g}\rangle=\left(\begin{array}{c}
\mathrm{e}^{x_{1}} \\
0 \\
\vdots \\
0
\end{array}\right)
$$

The commuting Hamiltonians for the open chain are obtained as coefficients of the characteristic polynomial

$$
\operatorname{det}(u-\mathcal{L})=A(u)
$$

respectively, for the quasiperiodic chain,

$$
\operatorname{det}(u-\mathcal{L}(v))=-v^{-1} \operatorname{det}(v-L(u)) .
$$

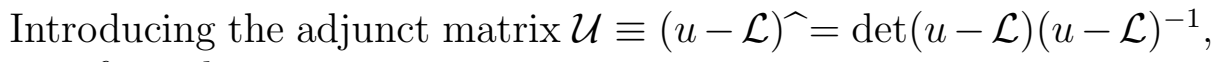
we get four identities:

$$
\begin{aligned}
\langle f|\mathcal{U}| g\rangle & =\mathcal{U}_{1 n}=1, \\
\langle\widetilde{f}|\mathcal{U}| g\rangle & =\mathrm{e}^{-x_{n}} \mathcal{U}_{n n}=C(u), \\
\langle f|\mathcal{U}| \widetilde{g}\rangle & =\mathrm{e}^{x_{1}} \mathcal{U}_{11}=-B(u), \\
\langle\widetilde{f}|\mathcal{U}| \widetilde{g}\rangle & =\mathrm{e}^{x_{1 n}} \mathcal{U}_{n 1}=1 .
\end{aligned}
$$

\section{SOLVING INVERSE PROBLEM}

Problem. Express the original variables $\mathrm{e}^{ \pm x_{j}}, X_{j}$ in terms of the dual ones $\Lambda_{j}, \lambda_{j}$.

The problem was stated and the solution for $n=3$ was given by Kuznetsov [Kuz2002] who made an important observation that $\mathrm{e}^{ \pm x_{j}}$, $X_{j}$ are expressed rationally in $\Lambda_{j}, \lambda_{j}$. The full solution $\forall n$ was given by Babelon [Bab2003, Bab2004] who obtained it by analyzing the hyperelliptic spectral curve for the quasiperiodic chain. The solution given below leads to the same final formulae as in [Bab2003, Bab2004] but is simpler since we work only with the rational spectral curve from the very beginning.

Solution. Introduce two covectors:

$$
\begin{array}{ll}
\Phi(u) \equiv\langle f| \mathcal{U}=\left(\varphi_{1}(u), \ldots, \varphi_{n}(u)\right), & \varphi_{j}(u)=\mathcal{U}_{1 j}, \\
\widetilde{\Phi}(u) \equiv\langle\widetilde{f}| \mathcal{U}=\left(\widetilde{\varphi}_{1}(u), \ldots, \widetilde{\varphi}_{n}(u)\right), & \widetilde{\varphi}_{j}(u)=\mathrm{e}^{-x_{n}} \mathcal{U}_{n j},
\end{array}
$$

that provide an analog of Baker-Akhiezer function for two leaves of a rational Riemann surface [KV2000]. Using $2 \times 2$ matrices we obtain:

$$
\varphi_{j}(u)=A_{n, j+1}(u), \quad \widetilde{\varphi}_{j}(u)=C_{j 1}(u) .
$$

Properties of $\Phi \widetilde{\Phi}$ :

(i) $\Phi \widetilde{\Phi}$ are polynomials in $u$ of degree $\leq n-1$. 
(ii) Asymptotics as $u \rightarrow \infty$ :

$$
\begin{gathered}
\varphi_{j}(u)=u^{n-j}+u^{n-j-1}\left(X_{n}+\ldots+X_{j+1}\right)+O\left(u^{n-j-2}\right), \\
\text { in particular, } \varphi_{n}=1 \text {. Also, } \\
\widetilde{\varphi}_{j}(u)=\mathrm{e}^{-x_{j}} u^{j-1}+O\left(u^{j-2}\right)
\end{gathered}
$$

in particular, $\widetilde{\varphi}_{1}=\mathrm{e}^{-x_{1}}$.

(iii) Conjugation conditions at points $u=\lambda_{j}$ :

$$
\widetilde{\Phi}\left(\lambda_{k}\right)=\Phi\left(\lambda_{k}\right) \Lambda_{k}, \quad k=1, \ldots, n .
$$

The equations (3.4) are identical to Babelon's [Bab2004] equations, though our interpretation in terms of the rational spectral curve is quite different.

The properties (i) and (ii) are easily verified. To derive the conjugation conditions (iii) it is sufficient to show that the polynomials

$$
F_{j}(u) \equiv \widetilde{\varphi}_{j}(u)-\varphi_{j}(u) C(u)
$$

are divisible by $A(u) \forall j \in\{1, \ldots, n\}$. Using (3.2) and the shorthand notation

$L_{n, j+1} \equiv L^{\prime \prime}=\left(\begin{array}{ll}A^{\prime \prime} & B^{\prime \prime} \\ C^{\prime \prime} & D^{\prime \prime}\end{array}\right), \quad L_{j 1} \equiv L^{\prime}=\left(\begin{array}{ll}A^{\prime} & B^{\prime} \\ C^{\prime} & D^{\prime}\end{array}\right), \quad L_{n 1} \equiv L=L^{\prime \prime} L^{\prime}$

we obtain

$$
F_{j}(u)=C^{\prime}-A^{\prime \prime} C
$$

Multiplying the first term $C^{\prime}$ by $1=\operatorname{det} L^{\prime \prime}=A^{\prime \prime} D^{\prime \prime}-B^{\prime \prime} C^{\prime \prime}$, and substituting $C=C^{\prime \prime} A^{\prime}+D^{\prime \prime} C^{\prime}$ into the second term one obtains, after, expanding the brackets,

$$
\begin{aligned}
F_{j} & =\left(A^{\prime \prime} D^{\prime \prime}-B^{\prime \prime} C^{\prime \prime}\right) C^{\prime}-A^{\prime \prime}\left(C^{\prime \prime} A^{\prime}+D^{\prime \prime} C^{\prime}\right) \\
& =-C^{\prime \prime}\left(B^{\prime \prime} C^{\prime}+A^{\prime \prime} A^{\prime}\right) \\
& =-C^{\prime \prime} A .
\end{aligned}
$$

The same can be derived in terms of the matrix $\mathcal{U}$. By virtue of (2.29) and (3.1) we have

$$
F_{j}(u)=1 \cdot \widetilde{\varphi}_{j}(u)-\varphi_{j}(u) \cdot C(u)=\mathcal{U}_{1 n} \cdot \mathrm{e}^{-x_{n}} \mathcal{U}_{n j}-\mathcal{U}_{1 j} \cdot \mathrm{e}^{-x_{n}} \mathcal{U}_{n n}
$$

or

$$
-\mathrm{e}^{x_{n}} F_{j}=\mathcal{U}_{1 j} \mathcal{U}_{n n}-\mathcal{U}_{1 n} \mathcal{U}_{n j}=\left|\begin{array}{ll}
\mathcal{U}_{1 j} & \mathcal{U}_{1 n} \\
\mathcal{U}_{n j} & \mathcal{U}_{n n}
\end{array}\right| .
$$

It remains to quote a theorem on the divisibility of the minors of the adjunct matrix by the determinant of the original matrix [Gan1966] (Chap. 1, Sect. 4, formula (33)). 
The properties (i-iii) are sufficient to reconstruct $\Phi \widetilde{\Phi}$ from $\lambda \Lambda$. Indeed, introducing explicitly the coefficients of the polynomials $\varphi,(u)$, $\widetilde{\varphi}_{j}(u)$

$$
\begin{aligned}
& \varphi_{j}(u)=r_{0}^{(j)}+r_{1}^{(j)} u+\ldots+r_{n-j-1}^{(j)} u^{n-j-1}+u^{n-j}, \\
& \widetilde{\varphi}_{j}(u)=q_{0}^{(j)}+q_{1}^{(j)} u+\ldots+q_{j-1}^{(j)} u^{j-1},
\end{aligned}
$$

we obtain for them a system of equations that follows from the conjugation conditions (3.4)

$$
q_{0}^{(j)}+\ldots+q_{j-1}^{(j)} \lambda_{k}^{j-1}-r_{0}^{(j)} \Lambda_{k}-\ldots-r_{n-j-1}^{(j)} \lambda_{k}^{n-j-1} \Lambda_{k}=\lambda_{k}^{n-j} \Lambda_{k},
$$

for $j=1, \ldots, n$.

The solution for $q_{m}^{(j)}$ and $r_{m}^{(j)}$ can be found by Cramer's rule. Then the rational formulae for $\mathrm{e}^{-x_{j}}$ and $X_{n}+\ldots+X_{j+1}$ in terms of $(\Lambda \lambda)$ can be extracted from the asymptotics (3.3). See [Bab2003, Bab2004] for the details. The commuting quantities $\mathrm{e}^{-x_{j}}$ provide thus the Hamiltonians for the dual (bispectral) problem.

\section{Quantisation}

Our treatment of the quantum case completely parallels the classical one. It is more convenient to work with the $2 \times 2$ Lax matrix rather then with the $n \times n$ one.

The Poisson brackets (2.1) are replaced with the commutation relations

$$
\left[X_{j}, \mathrm{e}^{ \pm x_{k}}\right]=\mp \mathrm{i} \eta \mathrm{e}^{ \pm x_{k}}
$$

the deformation parameter $\eta$ playing the role of the Planck constant.

The local Lax matrices $\ell_{j}(u)$ as well as the monodromy matrices $L_{j k}(u)$ and $L(u)$ are defined by the same formulae (2.3), (2.4), and (2.5) as in the classical case.

The classical $r$-matrix relations (2.16) and (2.17) are replaced with the quantum ones [Skl1985, Skl2000]

$$
\begin{gathered}
R\left(u_{12}\right) \stackrel{1}{\ell}\left(u_{1}\right) \stackrel{2}{\ell}\left(u_{2}\right)=\stackrel{2}{\ell}\left(u_{2}\right) \stackrel{1}{\ell}\left(u_{1}\right) R\left(u_{12}\right), \quad R(u)=u+\mathrm{i} \eta \mathcal{P}_{12}, \\
R\left(u_{12}\right) \stackrel{1}{L}_{j k}\left(u_{1}\right) \stackrel{2}{L_{j k}}\left(u_{2}\right)=\stackrel{2}{L}_{j k}\left(u_{2}\right) \stackrel{1}{L_{j k}}\left(u_{1}\right) R\left(u_{12}\right) .
\end{gathered}
$$

Note also the quantum determinant relation

$$
\mathrm{q}-\operatorname{det} T(u) \equiv A(u-\mathrm{i} \eta) D(u)-C(u-\mathrm{i} \eta) B(u)=1
$$

Since we work only with the open chain, all we need is the ACsubalgebra of (4.3)

$$
\begin{gathered}
{\left[A\left(u_{1}\right), A\left(u_{2}\right)\right]=\left[C\left(u_{1}\right), C\left(u_{2}\right)\right]=0,} \\
(u-v) A(u) C(v)+\mathrm{i} \eta C(u) A(v)=(u-v+\mathrm{i} \eta) C(v) A(u)
\end{gathered}
$$

Note that the above relations also hold for the partial monodromy matrix entries $A_{j k}$ and $C_{j k}$. 
Following [Skl1985] we define $\lambda_{j}$ as the zeroes of the self-commuting operator-valued polynomial $A(u)$ :

$$
A\left(\lambda_{j}\right)=0, \quad j=1, \ldots, n .
$$

Define for the operator-valued polynomial $F(u)$ the "substitution from the right" as

$$
F(u)=\sum_{m=0}^{p} F_{m} u^{m} \Longrightarrow[F(u)]_{u=\lambda_{k}} \equiv \sum_{m=0}^{p} F_{m} \lambda_{k}^{m} .
$$

Note that in [Skl1985] the "substitution from the left" is used instead but it makes only a little change for the calculations.

Using (4.8) define $\Lambda_{j}$ as

$$
\Lambda_{k}=[C(u)]_{u=\lambda_{k}},
$$

The quantum interpolation formulae are identical to the classical ones (2.22), one only needs to preserve the exact ordering of the operators as shown in (2.22b)

Using the same argument as in [Skl1985] one derives from (4.5) and (4.6) the commutation relations

$$
\begin{aligned}
& {\left[\lambda_{j}, \lambda_{k}\right]=\left[\Lambda_{j}, \Lambda_{k}\right]=0,} \\
& \Lambda_{j} \lambda_{k}=\left(\lambda_{k}+\mathrm{i} \eta \delta_{j k}\right) \Lambda_{j}
\end{aligned}
$$

replacing the classical Poisson brackets (2.21). For example, substituting $v=\lambda_{j}$ into (4.6) from the right one obtains

$$
A(u) \Lambda_{j}\left(u-\lambda_{j}\right)=\Lambda_{j} A(u)\left(u-\lambda_{j}+\mathrm{i} \eta\right)
$$

whence (4.10b) follows after substituting (2.22a), like in [Skl1985].

To solve the inverse problem in the quantum case we define the covectors $\Phi \widetilde{\Phi}$ by the same formulae (3.2) as in the classical case. Then we verify the same properties (i)-(iii) as in section 2 . The properties (i)(ii) being as trivial as in the classical case, we concentrate on proving (iii). Note that the order of operators in the conjugation conditions is now important, so (3.4) is now replaced with

$$
\left[\widetilde{\varphi}_{j}(u)\right]_{u=\lambda_{k}}=\left[\varphi_{j}(u)\right]_{u=\lambda_{k}} \Lambda_{k}, \quad \forall j, k
$$

or, using $\widetilde{\varphi}_{j}(u)=C^{\prime}(u) \equiv C_{j, 1}(u)$ and $\varphi_{j}(u)=A^{\prime \prime}(u) \equiv A_{n, j+1}(u)$,

$$
\left[C^{\prime}(u)\right]_{u=\lambda_{k}}=\left[A^{\prime \prime}(u)\right]_{u=\lambda_{k}} \Lambda_{k}, \quad \forall j, k .
$$

Amazingly, the derivation for the classical case has to be only slightly modified to be adapted to the quantum case.

Replace the classical formula (3.7) with

$$
F_{j}(u) \equiv 1 \cdot C^{\prime}(u)-A^{\prime \prime}(u-\mathrm{i} \eta) \cdot C(u) .
$$


The proof of (iii) is then given by the following chain of equalities

$$
\begin{aligned}
F_{j}(u)= & \left(A^{\prime \prime}(u-\mathrm{i} \eta) D^{\prime \prime}(u)-C^{\prime \prime}(u-\mathrm{i} \eta) B^{\prime \prime}(u)\right) \cdot C^{\prime}(u) \\
& -A^{\prime \prime}(u-\mathrm{i} \eta) \cdot\left(C^{\prime \prime}(u) A^{\prime}(u)+D^{\prime \prime}(u) C^{\prime}(u)\right) \\
= & \left.-C^{\prime \prime}(u-\mathrm{i} \eta) B^{\prime \prime}(u)\right) C^{\prime}(u)-A^{\prime \prime}(u-\mathrm{i} \eta) C^{\prime \prime}(u) \cdot A^{\prime}(u) \\
= & -C^{\prime \prime}(u-\mathrm{i} \eta) A(u) .
\end{aligned}
$$

using only the quantum determinant formula (4.4) and the identity

$$
A(u-\mathrm{i} \eta) C(u)=C(u-\mathrm{i} \eta) A(u)
$$

that follows from (4.6) for $u-v=-\mathrm{i} \eta$.

As soon as the properties (i)-(iii) are established, the rest is reduced to solving the systems of linear equations for the coefficients of $\Phi \widetilde{\Phi}$. For the details see [Bab2003, Bab2004]. The commuting quantities $\mathrm{e}^{-x_{j}}$ are expressed as finite-difference operators in $\lambda_{k}$ with rational coefficients, providing thus the Hamiltonians for the dual (bispectral) problem.

\section{Discussion}

Our improvement of the original derivation by [Bab2003, Bab2004] is twofold. In the classical case, we stress the use of the rational spectral curve, without recurse to the complicated hyperelliptic algebraic geometry. In the quantum case, we rely solely on the $R$-matrix algebra (4.3), without recurse to the integral representations for the eigenfunctions. As a consequence, our approach must also work for other integrable models of $R$-matrix type, like relativistic Toda chain, or XXX and XXZ spin chains.

Our approach to solving the inverse problem can also be used in the analysis of correlation functions for the Toda lattice [Koz2013].

\section{ACKNOWLEDGEMENTS}

The work is supported by EPSRC grant EP/H000054/1. I am grateful to Karol Kozłowski for discussions and for letting me know of his article [Koz2013].

\section{REFERENCES}

[Bab2003] O. Babelon, Equations in dual variables for Whittaker functions, Lett. Math. Phys. 65 (2003) 229-240

[Bab2004] O. Babelon, On the quantum inverse problem for the closed Toda chain, J. Phys. A: Math. Gen. 37 (2004) 303-316

[Chal2000] O. Chalykh, Bispectrality for quantum Ruijsenaars model and its integrable deformations, J. Math. Phys. 41 (2000) 5139-5167

[DG1986] J.J. Duistermaat, F.A. Grünbaum, Differential equations in the spectral parameter, Comm. Math. Phys.103 (1986) 177-240

[Fl1974] H. Flaschka, The Toda lattice, I. Existence of integrals, Phys. Rev. B9 (1974) 1924-1925

[Gan1966] F. R. Gantmakher, Theory of Matrices, Chesea Publ.:NY (1959) 
[KL2001] S. Kharchev, D. Lebedev, Integral representations for the eigenfunctions of quantum open and periodic Toda chains from the QISM formalism, $J$. Phys. A: Math. Gen. 34 (2001) 2247-2258

[Koz2013] K.K. Kozlowski, Aspects of the inverse problem for the Toda chain, in preparation

[KV2000] I. Krichever, K.L. Vaninsky, The periodic and open Toda lattice, - In: Mirror Symmetry IV, AMS/IP. vol. 33, 139-158 (2002)

[Kuz2002] V. Kuznetsov, Inverse problem for sl(2) lattices, - In: Symmetry and Perturbation Theory, World Scientific (2002), 136-152

[Man1974] S.V. Manakov, Complete integrability and stochastization of discrete dynamical systems, Zh. Exp. Teor. Fiz. 67 (1974) 543-555 [Russian]; English transl. in Sov. Phys. JETP 40 (1975) 269-274

[MTV2008] E. Mukhin, V. Tarasov, A. Varchenko, Bispectral and $\left(g l_{N}, g l_{M}\right) \mathrm{du}-$ alities, discrete versus differential, Adv. Math. 218 (2008) 216-265

[Skl1985] E. Sklyanin, The quantum Toda chain. - In: "Non-linear equations in classical and quantum field theory". Ed. by N. Sanchez. (Lecture Notes in Physics, vol. 226), N.Y.: Springer, 1985, p.196-233.

[Skl2000] E. Sklyanin, Bäcklund transformations and Baxter's Q-operator. - In: "Integrable Systems: From Classical to Quantum", eds. J. Harnad, G. Sabidussi and P. Winternitz, Centre de Recherches Mathématiques, Université de Montréal, CRM Proceedings and Lecture Notes, vol. 26 (2000), pp. 227-250

[TV2002] V. Tarasov, A. Varchenko, Duality for KnizhnikZamolodchikov and dynamical equations Acta Appl. Math. 73 (2002) 141-154 